\title{
Location Based Augmented Reality Application
}

\author{
Rachana Visavadiya $^{\mathrm{a}}$, Zunaira Zubair Ahmed ${ }^{\mathrm{a}}$
}

Augmented reality is a technology wherein the real-world surroundings are modified by information such as text, graphics, video or GPS data, which is generated digitally. This technology works by enhancing the user's current perception of the reality. Information about the real-world and its objects can be overlaid on the real-world. For adequate user experience, the displayed information must be specific. With advancement in AR technology, applications have been developed that make the user's environment interactive and, digitally alterable. In this paper, we describe the implementation of location-based augmented reality applications. Further information has been incorporated about the software that can be used to develop such an application.

Keywords: Augmented Reality; AR; GPS; QR; Unity; Location; application.

\section{Introduction}

Augmented reality (AR) refers to a type of virtual reality that enhances the environment through specific software and displays. It turns the surroundings of the user into a digital interface by placing virtual objects on the display in real-time.

The virtual objects include computer-generated input such as graphics, GPS data, sound, or video. As a result, the technology functions by enhancing one's current perception of reality. The development of this technology has been prompted by video games and mobile devices [1].

The main types of augmented reality are:

- Recognition: It includes object recognition and face recognition to provide additional information to the user in real-time. This type of AR is the most widely used one.

- Location: It uses GPS data to provide users with relevant information. One can use this type of application to determine their location and know the direction of where they need to go.

- Sensor: It is an object used to detect changes in its environment. The position and orientation are determined by combining data from the camera and an Inertial Measurement Unit (IMU).

- Outline: It merges the outline of some real-world object with virtual objects, allowing the user to identify and manipulate objects that may not exist in reality.

- Projection: It uses virtual imagery to augment what you see live. It projects digital images on physical objects in the existing physical space [1].

These may be marker-based or markerless AR. In markerbased $\mathrm{AR}$, the images or descriptors for augmentation are provided beforehand, for example, scanning barcodes or $\mathrm{QR}$ codes. For markerless AR, the images are not provided in advance. This becomes more difficult to implement, as the algorithm in the application must recognize patterns and other characteristics.

Augmented Reality not only possesses the ability to include objects to a real environment but also to remove them.
Ample focus has been given to the addition of virtual objects to the real environment in current work. However, graphic overlays may also be applied to hide or remove portions of the real environment from a user.

\section{TYPES OF APPROACH}

\section{A. Pattern recognition}

The software has to recognize a particular element which triggers the virtual information to be displayed. The element could be symbols, logos, or $\mathrm{QR}$ code. Image processing techniques are used to compare the image with the information stored in the database. The markers are designed in such a way that they can be recognized easily in the environment. The markers can be cylindrical targets, image targets, word or userdefined targets, 2D image frames (frame markers), Cloud targets, or virtual buttons (that can trigger an event). The content is a $2 \mathrm{D} / 3 \mathrm{D}$ object or video which we want to augment on top of a target.

\section{B. Location based}

This approach is used usually for GPS related applications, such as a city map showing restaurants on it using the camera. It does not need an external reference, like a specific logo, and it can be used worldwide.

The process of discerning the natural features of an environment to determine the location of the user can be computationally intensive. The present day smartphones are a favorable platform for developing AR applications as they are equipped with a camera, GPS and geomagnetic sensors, and internet access. The GPS and geomagnetic sensors in modern smartphones require lesser processing power and can operate in the environment without the need of specific markers. When users move their devices, the augmented information must immediately accommodate the modifications in the orientation and position of the device. Thus, sufficient knowledge about the surrounding environment should be gathered in a database to ensure satisfactory working of the application.

Additionally, new software can be delivered without many complications.

\section{IMPLEMENTATION}

Typical AR implementation contains two main parts: 
A. "Live" data

This is

augmented.

\section{B. "Meta" data}

This is used for the augmentation.

\section{PREVIOUS RESEARCH}

In 2010, "Location-based augmented reality on mobile phones" by R'emi Paucher and Matthew Turk was published in the IEEE Computer Society Conference. To support augmented reality applications on a mobile platform, this paper presents an approach to indoor localization. The position and orientation of the device can be known using the embedded camera.

Given that there are two approaches for augmentation i.e. pose computation and object recognition, the better approach is to determine the $6 \mathrm{DOF}$ (Degree of Freedom) pose of the sensor. Any 3D object from a database can be projected onto the image the user is viewing. Since there are many images of the environment in the database, it has been mentioned that an algorithm is required to select the appropriate image, with respect to the view of the user.

Database building is the first step in developing the application, obtaining data about the environment. The next step involves using cell phone sensors and pose estimation, once the indoor environment is suitable for location-based augmented reality. This requires working with the position and orientation of the device.

For the application to work as intended, a few criteria have been considered. When searching for an image in the database, it is not feasible to search all the possible images. Also, the image center has to be seen by the camera of the phone to avoid bad matching. To retrieve the correct image, its features were matched with the nearest neighbor feature of the database image. After a set of candidate images have been chosen, the unwanted were removed using an algorithm.

In the pose estimation process, three methods have been explained: reprojection minimization, first initialization method, and second initialization method. Out of these, the second initialization method was found to be faster, and provide better results.

For the tests, 3D rectangles as the virtual objects for ease of visualization and error measurement. The application was able to find the location of the device in the environment. The implementation was done in Symbian $\mathrm{C}++$ and Open $\mathrm{C} / \mathrm{C}++$ on a Nokia N97 cell phone. The computation time was more due to the SURF algorithm, and it does not support floatingpoint numbers.

In conclusion, it has been stated that the accuracy could be improved by improving the quality of the images.

In 2014, a paper titled "Location-based Mobile Augmented Reality Applications - Challenges, Examples, Lessons

Learned" was written and published by Philip Geiger, Marc Schickler, Rudiger Pryss, Johannes Schobel, Manfred Reichert of the University of Ulm. This paper gives information about design and implementation of a location-based augmented reality application on iOS and Andriod.

The development of a location-based mobile augmented reality engine has been described, and it is denoted as AREA (Augmented Reality Engine Application). The fundamental concept used in AREA is the locationView. The points of interest inside the camera's field of view are displayed on it, having a size of $\sqrt{ }_{\text {width }} 2+$ height 2 pixels. The locationView is placed centrally on the screen of the mobile device.

The points of interests (POIs) would be displayed accurately by AREA, even if the device is held obliquely. The camera's view of surrounding is not distorted as the locationView has been placed centrally on the screen. The POIs which were already drawn on the locationView can be reused, thus making its performance better.

The architecture of AREA has been designed in such a way, so as to be able to change and extend its components with ease. It consists of four main modules organized in a multi-tier architecture.

While implementing, in both iOS and Android, when a new location was detected, the POIs in the surrounding area of the user were to be determined by an adjustable radius. The basic software architecture of AREA on both mobile operating systems is same, with some differences in their implementation.

Since iOS and Android mobile operating systems are frequently updated, respective updates must be carefully considered when developing and deploying an advanced mobile business application like AREA.

\section{SOFTWARE IN USE TO DEVELOP AR APPLICATIONS}

\section{A. Vuforia}

Vuforia is an AR Software Development Kit (SDK) for mobile devices which facilitates the development of AR applications. It has a variety of recognition capabilities, some of which are:

- Image recognition: It represents images that the SDK can identify and track. The features that already exist in the image are tracked against a known target database. Device database and Cloud database are supported. The image will be tracked as long as it is in the camera's scope of view. It can be used to augment printed media for marketing, in gaming, etc. [2].

- Object Recognition: It enables the user to detect and track 3D objects. It has been designed to work on toys (such as action figures), and other consumer products. This feature can be used to develop an interactive experience with 3D objects [3].

- Text Recognition: Words can be detected that belong to an existing word list. The SDK provides a list of 100,000 commonly used English words which can be incorporated into an application. The developer can also define custom words, lists, and filters for blocking words from being recognized. Text recognition can be used separately, or in conjunction with object recognition [4]. 
- Extended Tracking: It uses features of the environment to enhance the tracking performance and maintain tracking even when the target is not in view anymore. As the target moves out of view, Vuforia utilizes other information from the surroundings to deduce the position of the target. A map is built around the target and presumes that both the environment and target are static. Using this feature, robust applications can be developed since the augmentations linked with the targets will remain [5].

- Smart Terrain: This feature is a vision capability that aids in creating a new level of AR gaming experience. It enables a developer to reconstruct the physical environment and create new kinds of visualization applications. The objects must have a static rigid and the surfaces must provide patterns which will be used the Smart Terrain Tracker to identify and track the object [6].

Vuforia provides Application Programming Interfaces (API) in $\mathrm{C}++$, Java, Objective-C++, and .Net languages through an extension to the Unity game engine. Thus, the SDK supports development for iOS and Android. Moreover, AR application can also be developed in Unity which can be exported to both platforms.

\section{B. Unity}

Unity is a cross-platform game engine with built-in IDE. It is mainly used for video game development and, allows for the user to develop in a generic environment provided by the software, and then build a solution for each of the possible platforms.

The game engine makes use of scripts for the interaction between elements. Unity includes an open-source implementation of the .NET framework called MonoDevelop for IDE. It is capable of interpreting JavaScript, C\#, and Boo(which has a Python-inspired syntax) [7].

There is full software package support which allows the developing of games. Features such as high frame rates, rapid iterative development, built-in support for many platforms, and collaboration with various device manufacturers makes Unity the foremost software to develop AR applications [8].

The main attributes for developing AR applications include libraries that interact with device sensors and give output in real-time. The use of Game Objects and scripts, along with simple deployment to a mobile device make Unity capable of creating AR applications. To develop a Unity AR project, there must be a camera scene to focus on the scene which acts as eyes of the user. There is a need for real-world positioning so that the device can read the location accurately. Unity supports a series of functions and classes for carrying out all these tasks [8].

\section{ACKNOWLEDGMENT}

We would like to sincerely thank our guide, Ms. Akrati Koul, for encouraging us to write this paper and for providing us with sufficient materials.

\section{CONCLUSION}

The mobile augmented reality is moving into the mainstream media at a quick rate. It finds applications in various fields ranging from gaming to tourism, to medical technologies.

Navigation applications using GPS systems make it easier to travel from one place to another, and can also be used to facilitate tourism. Tourists can walk through museums or historic sites and can see facts and figures about a specific display. This experience can also be enhanced by using applications that give a view of the history.

For medical applications, students can practice surgeries in a controlled environment using AR applications. Combining the applications with X-rays or MRI scans, surgeons can have everything in a single view.

Additionally, AR can be used by mechanics with a headworn display, to maintain and repair any machinery. AR technology can also be implemented in military, gaming, entertainment industry, as well as for advertising and promotions [9].

Augmented reality has been renewed recently due to the advance in mobile devices which complements the implementation of such applications. Advancements continue to take place in this field by researchers and manufacturers, making it an influential technology of the present and future.

\section{REFERENCES}

1. "Augmented Reality Games - Exciting Technology Around the Corner", Augmented-reality-games.com, 2017. [Online]. Available: http://www.augmented-realitygames.com/. [Accessed: 05- May- 2017].

2. "Image Targets | Vuforia Library", Library.vuforia.com, 2017.

[Online].Available:https://library.vuforia.com/articles/Trai ning/Im age-Target-Guide. [Accessed: 05- May- 2017].

3. "Object Recognition | Vuforia Library", Library.vuforia.com, 2017. [Online]. Available: https://library.vuforia.com/articles/training/objectrecognition. [Accessed: 05- May- 2017].

4. "Text Recognition | Vuforia Library", Library.vuforia.com, 2017.

[Online]. Available: https://library.vuforia.com/articles/Training/Text-RecognitionGuide.

[Accessed: 05- May- 2017].

5. Extended Tracking / Vuforia Library", Library.vuforia.com, 2017. [Online]. Available:

https://library.vuforia.com/articles/Training/ExtendedTracking. [Accessed: 05- May- 2017].

6. "Smart Terrain | Vuforia Library", Library.vuforia.com, 2017.

[Online]. Available: https://library.vuforia.com/articles/Training/GettingStarted-withSmart-Terrain. [Accessed: 05- May- 2017].

7. A. S. Font and D. R. Vall, "Master thesis," 2013.

8. "Unity - Unity - Multiplatform - VR-AR", Unity, 2017. [Online]. Available: https://unity3d.com/unity/multiplatform/vr-ar. [Accessed: 05- May- 2017].

9. "All You Wanted to Know about Augmented Reality", Lifewire, 2017. [Online]. Available: https://www.lifewire.com/applicationsof-augmentedreality-2495561. [Accessed: 05- May- 2017]. 
